
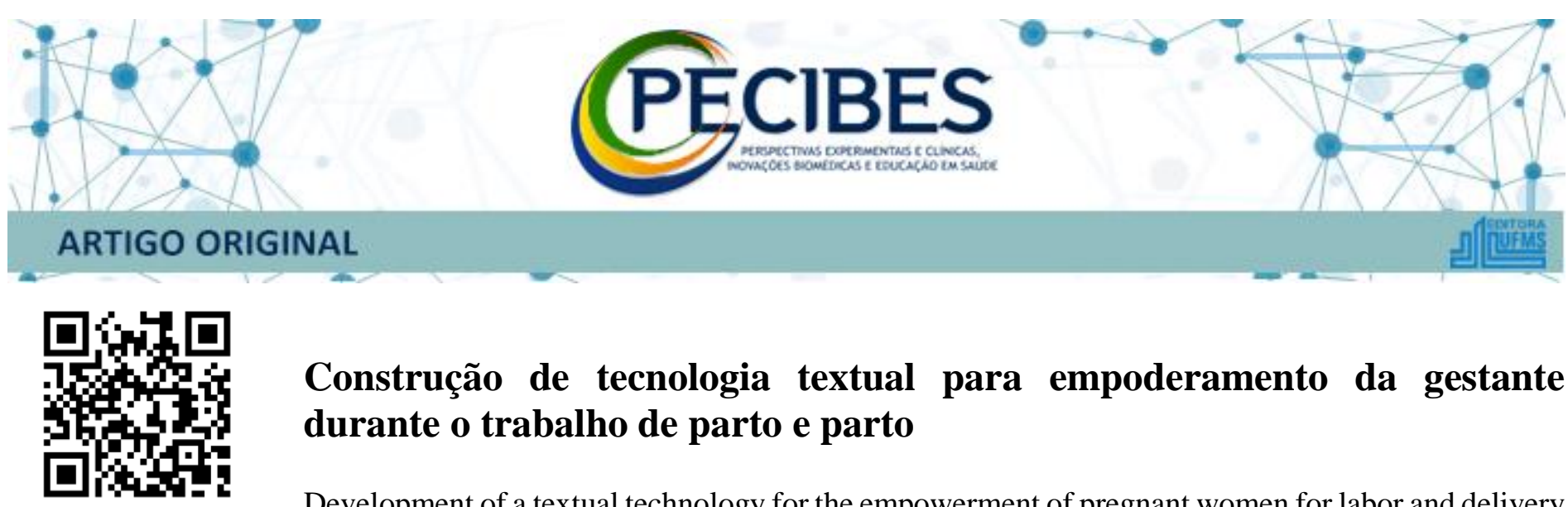

http://www.seer.uf ms.br/index.php/p ecibes/index

\title{
Construção de tecnologia textual para empoderamento da gestante durante o trabalho de parto e parto
}

Development of a textual technology for the empowerment of pregnant women for labor and delivery

\author{
Ana Carla Tamisari Pereira ${ }^{1}$, Marcelo Gonçalves da Silva ${ }^{2}$, Lourdes Missio ${ }^{2}$. \\ ${ }^{1}$ Hospital Universitário da Grande Dourados/EBSERH \\ ${ }^{2}$ Universidade Estadual de Mato Grosso do Sul - UEMS
}

\section{*Autor}

correspondente

:Ana Carla

Tamisari

Pereira E-mail: atamisari@ms

n.com

\section{Resumo}

Introdução: A educação em saúde pode ser entendida como um campo de teoria e prática interdisciplinar, voltado para autonomia do indivíduo, dando subsídios para que o mesmo possa ser corresponsável perante suas condições de saúde. Objetivo: descrever o processo de Construção de tecnologia textual para empoderamento da gestante durante o trabalho de parto e parto. Método: Trata-se de uma pesquisa de natureza exploratória e metodológica e teve como fundamentação teórica o referencial da Andrea Filatro. Resultados: O guia educativo foi construído tendo como referencial a teoria socioconstrutivista de Paulo freire. O processo de elaboração envolveu 04 etapas sendo: 1- busca das principais dúvidas das gestantes sobre o trabalho de parto e parto; 2- seleção do conteúdo teórico para compor o guia; 3- seleção e elaboração de imagens para ilustrar o material e 4- diagramação do material. Conclusão: Espera-se que este material seja utilizado pelas gestantes, atendidas na saúde pública e ou privada, e que auxilie nessa busca pelo conhecimento. Que esse conhecimento ajude na construção do seu empoderamento e, ao chegar à maternidade para dar à luz, tenha segurança, tranquilidade e, confiança para estarem abertas às novas práticas de assistência ao parto normal.

Palavras-chave: Educação em saúde. Empoderamento. Gravidez. Tecnologia. Parto

\begin{abstract}
Introduction: Health education can be understood as a field of interdisciplinary theory and practice, aimed at promoting the individual's autonomy, providing subsidies for them to be co-responsible for their health conditions. Objective: This study aims to describe the process of constructing a textual educational technology aimed at preparing pregnant women for childbirth. Method: This is an exploratory and methodological research and had as theoretical foundation Andrea Filatro's framework. Results: The educational guide was built using Paulo Freire's socio-constructivist theory as a reference. The elaboration process involved 04 steps: 1- search for the main doubts of pregnant women about labor and delivery; 2- selection of theoretical content to compose the guide; 3- selection and elaboration of images to illustrate the material and 4- layout of the material. Conclusion: It is expected that this material is used by pregnant women, both in the basic health network and in the private network, and that it helps in this search for knowledge. May this knowledge help in building their empowerment and, upon arriving at the maternity ward to have their child, that they have security, tranquility, and confidence to be open to new care practices for normal birth
\end{abstract}

Key-words: Health education. Empowerment. Pregnancy. Technology. 


\section{Introdução}

A educação em saúde pode ser entendida como um campo de teoria e prática interdisciplinar, voltado para promoção da autonomia do indivíduo, dando subsídios para que o mesmo possa ser corresponsável perante suas condições de saúde. Pode ser desenvolvida com ações pontuais ou não, desde que possibilite aos sujeitos ressignificar e coproduzir conhecimentos favorecendo a emancipação do cuidado ${ }^{1}$.

Diante da importância da educação em saúde para promover a autonomia do indivíduo perante seus cuidados com a saúde, sua a preparação para o parto tem como objetivo e encorajamento das grávidas e seus companheiros para a participação ativa no processo de nascimento. $\mathrm{O}$ momento da preparação envolve orientações sobre exercícios respiratórios para ajudar no controle da dor, orientações sobre as vias de parto e as diversas posições para o período expulsivo. As gestantes primigestas são as que mais procuram informações e participam em grupos de educação em saúde ${ }^{2}$.

Para tanto, as portarias e programas do Ministério da Saúde estimulam ações educativas, objetivando melhorar a saúde das gestantes. Nas ações do Programa de Humanização do Pré-natal e Nascimento, destaca-se a importância dessas atividades na perspectiva do empoderamento da mulher para o enfrentamento do parto $^{3 .}$

Partindo da premissa que a preparação para o parto é necessária, e no momento do parto não é a hora ideal para que a mulher receba muitas informações sobre o processo do trabalho de parto, esse deve ocorrer durante as consultas de pré-natal ou em atividades educativas vinculadas ao período ${ }^{4}$.
Portanto, promover ações de educação em saúde durante o ciclo gravídico-puerperal, por meio de grupos de gestantes, se faz necessário, principalmente enfatizando não só o cuidado humanizado, mas possibilitando o empoderamento da mulher e do seu acompanhante ${ }^{4}$.

A consulta de pré-natal com o enfermeiro, bem como as atividades educativas, oportuniza informar, aconselhar, educar e contribuir para a promoção da saúde. A interação entre o enfermeiro e a gestante permite a troca de saberes e de informações entre eles, aproveitando para instruir sobre os benefícios do parto normal, estimulá-la a ser protagonista do processo de nascimento e melhorar a qualidade da assistência prénatal ${ }^{5}$.

Segundo Couto $^{2}$, as mulheres que participaram de grupos de orientação para o parto, adquiriram maior conhecimento e confiança no momento de parir, e revelaram ter um trabalho de parto com menos dor. Diante disso, entre os fatores que se relacionam a uma forma mais positiva de vivenciar o parto, destacam-se o conhecimento e a confiança. Os grupos podem ser espaços importantes para aprender a lidar com os medos relativos ao nascimento ${ }^{2}$.

Os grupos de educação em saúde consistem num valioso instrumento terapêutico para promover discussões, aumentar o aprendizado, possibilitando estabelecer um vínculo entre a gestante e o profissional de saúde, dando oportunidade de melhorar a implementação do cuidado ${ }^{6}$.

Para o Ministério da Saúde, os métodos contemporâneos de educação preparatória para o parto tendem a combinar aspectos das diversas abordagens dos métodos de Dick-Read, Lamaze e Bradley. Esses métodos tendem a modificar a atitude da mulher e do seu 
acompanhante, e não mais ligar relacionar o parto ao medo e à dor, e sim aceitá-lo de forma compreensiva, com segurança, tranquilidade levando à-participar de forma ativa ${ }^{7}$.

Uma das formas de preparar a gestante para o parto é utilizar materiais impressos, cuja principal finalidade é divulgar conteúdos considerados essenciais para prevenção e tratamento de doenças, procurando reforçar orientações transmitidas durante as consultas e contribuir para a implementação do seu próprio cuidado. Uma das características dos materiais educativos é fazer a mediação entre os profissionais de saúde e a população ${ }^{8}$.

Diante da importância da preparação da gestante para enfrentamento do trabalho de parto e parto, este estudo tem como objetivo descrever o processo de construção de uma tecnologia educativa textual para preparação das gestantes para o parto.

\section{Metodologia}

Trata-se de uma pesquisa de natureza exploratória e metodológica que visou construir uma tecnologia textual, para ser utilizada com gestantes, visando seu empoderamento no a trabalho de parto e parto.

A pesquisa metodológica, segundo Lima ${ }^{9-16}$, "lida com o desenvolvimento, validação e avaliação de ferramentas e métodos de pesquisa".

Para Nascimento ${ }^{10-31}$,esse tipo de pesquisa:

Utiliza de maneira sistemática os conhecimentos existentes para elaborar uma nova intervenção ao ou melhora significativa de uma intervenção existente, ou ainda, elabora ou melhora um instrumento, um dispositivo ou um método de mediação.
A construção desta tecnologia textual, no formato de um guia, teve como fundamentação teórica o referencial descrito por Andrea Filatro ${ }^{11}$. De acordo com a autora, o conteúdo educativo textual no qual a mesma refere como mídia impressa, é o registro de um material físico, cujas matrizes de linguagem verbal e visual apresentam conteúdo que propõem atividades de aprendizagem nas mais diversas áreas do conhecimento $^{11}$.

A tecnologia textual impressa é um meio de fácil utilização, pois permite acesso rápido às informações. Nela, o leitor pode realizar pesquisas rápidas e consultar referenciais em tópicos específicos do seu interesse ${ }^{11}$.

O processo de elaboração deste material, que ora discutimos, envolveu quatro etapas sendo: 1- busca das principais dúvidas das gestantes sobre o trabalho de parto e parto; 2- seleção do conteúdo teórico para compor o guia; 3- seleção e elaboração de imagens para ilustrar o material e 4- diagramação do material.

Neste texto apresentaremos como a tecnologia foi desenvolvida.

A pesquisa atendeu os preceitos Éticos, de acordo com a Resolução n. 466/12 do Conselho Nacional de Saúde/Ministério da Saúde. O projeto foi aprovado pela Comissão de Avaliação em Pesquisa e Extensão (CAPE), do HU/UFGD, e pelo Comitê de Ética da Universidade Estadual do Mato Grosso do Sul, sob o parecer n. 2.315.422 e CAAE n. 73678217.3.0000.8030

\section{Resultados e Discussão}

Neste estudo, também se salienta a nossa prática assistencial em obstetrícia, na qual percebemos que as gestantes, ao se internarem para o parto, bem como seus 
acompanhantes, encontravam-se despreparadas, com medo e inseguras com relação ao trabalho de parto e parto. Sentindo a necessidade de empoderar essas mulheres, desenvolvemos um guia educativo com o objetivo de auxiliar a gestante e seu acompanhante para o enfrentamento do trabalho de parto e parto.

Para a construção do guia, utilizamos como referencial teórico a teoria socioconstrutivista de Paulo Freire. Suas obras trazem como mensagem principal, a libertação, a autonomia do indivíduo para que construa seu conhecimento, sua criticidade, seu empoderamento.

Assim, Freire trouxe ao Brasil, o significado da palavra empowerment, pois desenvolveu esse conceito no período que morou nos Estados Unidos e, junto com seu colega Ira Shor, escreveram o livro "Medo e Ousadia"12. Nesta obra, descrevem o que significaria esta palavra, dentro de uma cultura americana, na qual as minorias enfrentavam dificuldades para ingressar no nível superior, como os professores que tinham dificuldades de implementar um novo método pedagógico, devido as perseguições e ameaças que sofriam. Apontam que:

O conceito de empowerment, está ligado as classes sociais, como a classe trabalhadora, que através de suas próprias experiências, sua própria construção de cultura, seu empenho na obtenção de poder político ${ }^{12-72}$.

Dentro da teoria socioconstrutivista de Paulo Freire, a palavra empowerment é termo-chave para a promoção da saúde, no qual as mudanças sociais são o caminho para aquisição do conhecimento relativo à capacidade discursiva, cognitiva e processual. No processo educativo, o empoderamento acontece quando educadores e educandos se permitem construir o conhecimento juntos ${ }^{13} 14$.
Dessa forma, a filosofia educacional de Paulo Freire permitiu o empoderamento pelo mundo. Ele defende a conscientização como um ciclo contínuo de diálogo e ação, no qual o convívio entre as pessoas permite o diálogo e a reflexão, contribuindo para o desenvolvimento de uma ação coletiva e participativa que, por sua vez, gera novas reflexões e ações permitindo transformar o mundo ${ }^{13}$.

Partindo dessa premissa, para que essas gestantes sejam empoderadas, foi necessário verificar seu conhecimento prévio sobre o trabalho de parto e parto, e por meio dessas informações, elaborar um guia que as mesmas pudessem utilizar para resgatar sua autonomia, confiança, segurança, tranquilidade para o momento do parto.

Primeiramente, foi aplicado um questionário para conhecer as características sociodemográficas e obstétricas das gestantes. Logo após, foi realizada uma entrevista semiestruturada, individual e audiogravada para identificar o seu conhecimento sobre o trabalho de parto e parto e compreender seu empoderamento para enfrentar o processo de nascimento. As entrevistas foram gravadas com uso de um gravador digital. O tempo médio para aplicação do questionário e a entrevista foi de 30 a 40 minutos.

O conteúdo da tecnologia textual foi definido através da análise das entrevistas, utilizando o discurso sujeito coletivo de Lefevre, Lefevre ${ }^{15}$, a partir das dúvidas mais frequentes encontradas nas falas das mesmas. Segue alguns exemplos das dúvidas mais comuns relatadas pelas gestantes:

O medo. A minha primeira foi cesárea, então pra mim é tudo mais rápido a cesárea do que um parto normal, eu não passei pelo processo do parto normal. (E1) 
Seria mais a dúvida que eu tinha sobre sutura, né, que todo mundo fala que antes dava o pique, agora não dá, só essa dúvida mesmo que eu tinha mais medo assim. (E4)

[...] Tipo a hora que está chegando, tipo que hora que é pra ir pro hospital [...]. (E5)

Da contração né? Estoura a bolsa, a única coisa que eu sei. (E6)

Acho que é essa que eu falei abaixada, essa na bola que pode ajudar a estimular. (E7)

A primeira etapa da construção do guia foi buscar as principais dúvidas que serviram de pontos norteadores para construção do guia, sendo: desconhecimento sobre os sinais de trabalho de parto, a hora de ir para o hospital, o medo do corte da episiotomia, o medo do parto normal, o medo da dor de parto, os benefícios do parto normal para mulher e para o bebê, a indicação de cesariana, os métodos não farmacológicos para alíivio da dor durante o trabalho de parto, as posições para o parto normal e, também, sobre a atuação da enfermeira obstetra no parto normal de risco habitual.

A segunda etapa envolveu a seleção do conteúdo teórico para compor o guia. Foram selecionados materiais disponibilizados pelo Ministério da Saúde e OMS e, também, em trabalhos publicados sobre a temática. Procurou-se usar uma linguagem facilitadora, clara e de fácil entendimento.

O enfoque do conteúdo do guia iniciou com os sinais de trabalho de parto até o nascimento do bebê. Foi utilizada uma chamada para a gestante "A hora chegou!", propositalmente, para que ao chamar a atenção pudesse despertar o interesse da mesma para seguir lendo o material.

Assim, o guia foi desenvolvido em forma de perguntas e respostas, como se tivesse respondendo às principais inquietações das gestantes. As perguntas foram colocadas de forma cronológica ao processo dos períodos clínicos do parto, iniciando com os sinais de trabalho de parto e finalizando com o nascimento do bebê.

O título foi o último a ser pensado, porque precisava ser algo que contemplasse o objetivo da produção do guia, envolvendo o empoderamento da gestante. Diante de tantas dúvidas e incertezas denotadas nas falas das gestantes, durante as entrevistas, a escolha do título "O que esperar para a hora do nascimento" nos remete a pensar que, cada gestação é única, singular, com suas particularidades e, cada mulher não sabe o que esperar para a hora do nascimento. Um parto não é igual ao outro. Então, essa incerteza aguça sua curiosidade e desperta vontade de conhecer mais.

A terceira etapa foi a seleção das imagens, para tornar o material mais fácil de entender. Queríamos que as imagens falassem por si só. Assim, as ilustrações do guia educativo foram pensadas como algumas imagens de livros e, também, do quadro "Caminhando para o parto normal" disponibilizado nas maternidades públicas e da cartilha "Celebrando a Vida"16. Desta forma, as imagens foram utilizadas como modelo e desenhadas por um profissional habilitado. Para Echer ${ }^{16}$ é importante ilustrar as orientações para tornar a tecnologia compreensível e descontraída. As ilustrações podem tornar-se autoexplicativas.

Portanto, as imagens do guia buscam ilustrar o conteúdo teórico, de maneira a auxiliar no entendimento e facilitar a assimilação pela gestante. A imagem da capa 
foi selecionada para representar a gestante que espera nove meses para o nascimento do seu bebê, e o coração simboliza a vida em crescimento e o amor que é construído a cada dia pela mulher.

$\mathrm{Na}$ quarta etapa, após a seleção do conteúdo teórico e das imagens, foi contratado um profissional especializado, para a realização da diagramação, seguindo as recomendações da NBR número $6029^{17}$.

\section{Considerações Finais}

A teoria socioconstrutivista de Paulo Freire foi utilizada como referencial teórico para construção do guia educativo. Sua filosofia pedagógica permitiu o empoderamento das pessoas pelo mundo, na perspectiva de que o diálogo entre os educadores e educandos é capaz de produzir o conhecimento e sua reflexão crítica. Com as gestantes isso não é diferente, a partir do momento que elas recebem informações sobre aquilo que as causam dúvidas e medos, associam ao seu conhecimento prévio, e são capazes de refletir sobre o que é melhor para o nascimento do seu filho, de maneira que se sintam empoderadas para enfrentar aquela determinada situação.

Nessa perspectiva, esse guia educativo foi construído com o objetivo de auxiliar as gestantes e seus acompanhantes para enfrentarem o momento do trabalho de parto e parto, abordando as principais dúvidas que as mesmas têm para a hora do nascimento. Espera-se que este material seja utilizado pelas gestantes, tanto da rede básica de saúde quanto da rede privada, e que auxiliem nessa busca pelo conhecimento.

Consideramos esse conhecimento importante na construção do seu empoderamento para que, ao chegar à maternidade para o nascimento de ter seu filho, esteja aberta às novas práticas de assistência ao parto normal, com confiança, tranquilidade e segurança.

\section{Referências}

Flisch TMP, Alves RH, De Almeida TAC, De Torres HC, Schall VT, Dos Reis DC. Como os profissionais da atenção primária percebem e desenvolvem a Educação Popular em Saúde? Interface Commun Heal Educ [Internet]. 2014;18:1255-68. Disponível em : https://www.scielo.br/scielo.php?script=sci_artte $\mathrm{xt} \& \mathrm{pid}=\mathrm{S} 1414-32832014000601255$. Acesso em: $29 / 11 / 2021$

Couto GR. Conceitualização pelas enfermeiras de preparação para o parto. Rev Lat Am Enfermagem . 2006;14(2):190-8. Disponível em: https://www.scielo.br/scielo.php?script=sci_artte $\mathrm{xt} \& \mathrm{pid}=\mathrm{S} 1414-32832014000601255$. Acesso em: $29 / 11 / 2021$

Reberte LM, Hoga LAK, Gomes ALZ. O processo de construção de material educativo para a promoção da saúde da gestante. Rev Latino-Am Enferm. 2012;1(6):8. Disponível em: https://www.scielo.br/scielo.php?pid=S0104$11692012000100014 \&$ script=sci_abstract $\&$ tlng $=$ pt. Acesso em: 29/11/2021

Camillo BS, Nietsche EA, Salbego C, Cassenote LG, Dal Osto DS, Böck A. Ações de educação em saúde na atenção primária a gestantes e puérperas: revisão integrativa TT - Health education actions in primary attention to pregnant and puerperal women: integrative review. Rev enferm UFPE. 2016;10(6):4894-901.Disponívem em: http://www.revista.ufpe.br/revistaenfermagem/in dex.php/revista/article/view/8573/pdf_2024.

Acesso em: 29/11/2021

Guedes GW, Sousa MNA de, Lima TNF de A, Lima MNF de A, Davim RMB, Costa TS. Conhecimentos de gestantes quanto aos benefícios do parto normal na consulta pré-natal. Rev enferm UFPE. 2016;10(10):3860-7. Disponíel em: 
http://www.revista.ufpe.br/revistaenfermagem/index.ph p/revista/article/viewFile/10107/pdf_11264.

Acesso em: 29/11/2021

Alves FLC, Castro EM, Souza FKR, Lira MCP de S, Rodrigues FLS, Pereira L de P. Grupo de gestantes de alto-risco como estratégia de educação em saúde. Rev Gauch Enferm. 2019;40:e20180023. Disponível em: https://www.scielo.br/pdf/rgenf/v40/1983-1447rgenf-40-e20180023.pdf. Acesso em: 29/11/2021

Brasil. Ministério da Saúde. Secretaria de Políticas de Saúde. Área Técnica de Saúde da Mulher. Parto, Aborto e Puerpério: Assistência Humanizada à Mulher. Vol. 13, Ministério da Saúde. Brasília; 2001. 44-55 p. Disponível em: http://www.sciencedirect.com/science/article/pii/ S0104423011704055\%5Cnhttp://www.revistas.u sp.br/rlae/article/view/2392\%5Cnhttp://www.sci elo.br/scielo.php?script=sci_arttext\&pid=S0100$72032014000400152 \& \operatorname{lng}=$ en $\& n r m=i s o \& \operatorname{lng}=p$ t\%5Cnhttp://pepsic.bvsalud.org/s. Acesso em: $29 / 11 / 2021$

Freitas FV de, Rezende Filho LA. Modelos de comunicação e uso de impressos na educação em saúde: uma pesquisa bibliográfica. Interface Comun Saúde, Educ. 2010;15(36):243-56. Disponível em: https://www.scielo.br/scielo.php?pid=S141432832010005000044\&script=sci_abstract\&tlng= pt. Acesso em: 29/11/2021

Lima DVM de. Diseños de pesquisa: una contribución al autor. Online Brazilian J Nurs. 2011;3648. Disponível em: http://www.objnursing.uff.br/index.php/nursing/ article/viewFile/3648/pdf_2. Acesso em: 29/11/2021

Malheiros PA, Alves VH, Rangel TSA, Vargens OM da C. Parto e nascimento: Saberes e práticas humanizadas. Texto e Context Enferm. 2012;21(2):329-37.

Filatro A, Bileski SMC. Produção de conteúdos educacionais: design instrucional, tecnologia, gestão, educação e comunicação. Saraiva. Saraiva, editor. São Paulo: Saraiva; 2015. 480 p.

Shor I, Freire P. Medo e ousadia: o cotidiano do professor. Vol. 18, Paz e Terra. Rio de Janeiro; 1986. $562 \mathrm{p}$.

Freire P. Pedagogia do Oprimido. Rio de Janeiro: Paz e Terra; 1987. 129 p.

Freire P. Pedagogia da Autonomia: saberes necessários à prática educativa. 25th ed. Rio de Janeiro: Paz e Terra; 1996. 54 p.

Lefrève F, Lefrève AMC. O discurso do sujeito coletivo - um novo enfoque em pesquisa qualitativa (desdobramentos). EDUCS. Caxias do Sul: EDUCS; 2020. 138 p.

REBERTE, Luciana Magnoni; HOGA LAK. Celebrando a vida: construção de uma cartilha para promoção da saúde da gestante. Univ São Paulo [Internet]. 2008;130. Disponível em: http://www.teses.usp.br/teses/disponiveis/7/7132 /tde-05052009-112542/. Acesso em: 29/11/2021.

Echer IC. Elaboração de manuais de orientação para o cuidado em saúde. Assoc Psychol Sci [Internet]. 2005;13(5):754-7. Disponível em: https://www.psychologicalscience.org/news/rele ases/supporting-patient-autonomy-is-critical-toimproving-health.html. Acesso em: Acesso em: $29 / 11 / 2021$

TÉCNICAS ABDN. NBR 6029: informação $e$ documentação - livros e folhetos - apresentação. Assoc Bras NORMAS TÉCNICAS. 2006; 